\title{
Assessment of damage and anisotropic plasticity models to predict Ti-6Al-4V behavior
}

\author{
C. F. Guzmán ${ }^{1, \mathrm{a}}$, V. Tuninetti ${ }^{1, \mathrm{~b}}$, G. Gilles ${ }^{1, \mathrm{c}}$, A. M. Habraken ${ }^{1,2, \mathrm{~d}}$ \\ ${ }^{1}$ ArGEnCo Department, MS²F Division, University of Liège, Chemin des Chevreuils 1, 4000 Liège, \\ Belgium \\ ${ }^{2}$ Research Director of the Fonds de la Recherche Scientifique - FNRS, Belgium \\ acf.guzman@ulg.ac.be, bvictor.tuninetti@gmail.com, 'cggilles@ulg.ac.be, \\ danne.habraken@ulg.ac.be
}

Keywords: Ductile fracture; GTN model; Void nucleation; Void growth; Void coalescence; CPB06 yield criterion; anisotropy.

\begin{abstract}
The plastic behavior of the Ti-6Al-4V alloy includes several features as strength differential effect, anisotropy and yield strength sensitivity to temperature and strain rate. Monotonic tensions in the three orthogonal directions of the material are performed to identify the Hill ' 48 yield criterion. Monotonic compression and plane strain tensile tests are also included in the experimental campaign to identify the orthotropic yield criterion of CPB06. An assessment of the two models is done by comparing the yield loci and the experimental data points for different levels of plastic work. A first approach of the damage modelling of the Ti-6AL-4V alloy is investigated with an extended GursonTvergaard-Needleman damage model based on Hill ' 48 yield criterion. Finite element simulations of the experiments are performed and numerical results allows checking force-displacement curves until rupture and local information like displacement and strain fields. The prediction ability of the Hill '48, CPB and extended Gurson models are assessed on simple shear and notched tensile tests until fracture.
\end{abstract}

\section{Introduction}

Aerospace and manufacturing industries have a strong interest in numerically assessment of the ductile fracture of Ti-6Al-4V in real components. Indeed the formability and crashworthiness of Ti-6AL-4V are frequent limitations due to the material ductile fracture.

As for most metals and alloys, Ti-6AL-4V shows a process of material degradation including three successive stages of damage mechanism: nucleation, growth and coalescence of voids. Note that the first two mechanisms may occur simultaneously. After nucleation, the voids resulting from interface decohesion of inclusions or particle fracture grow within the surrounding matrix and affect its plastic deformation. The first stage of void growth by relatively homogeneous plastic deformation of the surrounding matrix is interrupted by the localization of the plastic flow in the ligament between the voids. This localization corresponds to the onset of coalescence.

There are currently several damage models avaible in the literature in order to describe the material degradation. Two categories can be distinguished: the phenomenological (e.g., Lemaitre [1]) and the micromechanical approaches. The most popular micromechanical model for ductile damage is the Gurson-Tvergaard-Needleman (GTN). Gurson [2] first proposed a porous plasticity model with the void volume fraction as internal variable. The model was further improved to account the loss of load carrying capacity associated with void nucleation and coalescence [3], hence considering the three stages of damage: void nucleation, growth and coalescence. Since then, the GTN model has been extended to cover different applications. For instance, Benzerga and Besson [4] performed the same analysis as Gurson [2] but considering an anisotropic matrix of the Hill' 48 type [5]. A fully-implicit integration scheme was proposed by Ben Bettaieb et al. [6] for this anisotropic GTN model, also including the effect of the kinematic hardening. 
The main goal of this present paper is to calibrate the GTN model on bulk Ti-6AL-4V alloy at room temperature and a quasi-static strain rate and to check its ability to predict fracture. In addition, the load predictions of three different models applied on notched round bars and holed specimens are assessed. The first model is based on the Hill '48 [5] yield criteria, which takes into account the anisotropic behavior of Ti-6AL-4V alloy. The second model is defined by the orthotropic yield criterion CPB06 that accounts for both the anisotropy and the strength differential effect of the alloy [7]. The third one predicts damage by the extended version of the GTN model.

\section{Material models}

Three different material models are used in the article. In the case of the Hill '48 and the GTN model, the used a Voce type of isotropic hardening law.

$$
\sigma_{Y}=\sigma_{0}+K\left(1-\exp \left(-n \epsilon_{e q}^{P}\right)\right)
$$

where $\sigma_{Y}$ is the flow stress, $\epsilon_{e q}^{P}$ is the equivalent plastic strain and $K, n, \sigma_{0}$ are material constants computed from a tensile curve in a reference direction. The CPB06 model uses also the Voce law as a reference hardening curve [7].

Hill ' 48 model. In the yield criteria introduced by Hill [5] the material has three orthogonal symmetry planes and three principal axes of anisotropy: $x, y$ and $z$. In terms of the anisotropic axis:

$$
\begin{aligned}
& F_{p}\left(\sigma_{i j}\right)=\frac{1}{2}\left[F\left(\sigma_{y y}-\sigma_{z z}\right)^{2}+G\left(\sigma_{z z}-\sigma_{x x}\right)^{2}+H\left(\sigma_{x x}-\sigma_{y y}\right)^{2}\right. \\
& \left.+2 L{\sigma_{y z}}^{2}+2 M \sigma_{z x}^{2}+2 N{\sigma_{x y}}^{2}\right]-{\sigma_{Y}}^{2}=0
\end{aligned}
$$

where $F, G, H, L, M, N$ are material parameters. Note that if $F=G=H=1.0$ and $L=M=$ $N=3.0$, the Hill '48 yield locus recovers the isotropic von Mises yield locus.

The CPB06 model. The orthotropic yield criterion CPB06 was developed by Cazacu et al. [8]. The criterion is defined by:

$$
F_{p}\left(\sigma_{i j}\right)=\left(\left|\Sigma_{1}\right|-k \Sigma_{1}\right)^{a}+\left(\left|\Sigma_{2}\right|-k \Sigma_{2}\right)^{a}+\left(\left|\Sigma_{3}\right|-k \Sigma_{3}\right)^{a}
$$

where $k$ is a parameter which takes into account the strength differential (SD) effect and $a$ is the degree of homogeneity. $\Sigma_{1}, \Sigma_{2}, \Sigma_{3}$ are the principal values of the second-order tensor $\Sigma_{i j}=C_{i j k l}: S_{k l}$, where $C_{i j k l}$ is a fourth-order orthotropic tensor that accounts for the plastic anisotropy of the material and $S_{i j}$ is the deviatoric part of the Cauchy stress tensor.

The GTN yield surface. The classical GTN yield surface is defined by:

$$
F_{p}\left(\sigma_{i j}, f, \sigma_{Y}\right)=\frac{\sigma_{e q}^{2}}{\sigma_{Y}^{2}}-1+2 q_{1} f \cosh \left(-\frac{3 q_{2}}{2} \frac{\sigma_{m}}{\sigma_{Y}}\right)-\left(q_{1} f\right)^{2}=0
$$

where $\sigma_{e q}$ is the (macroscopic) equivalent stress related to Hill '48 yield criterion, $\sigma_{Y}$ the yield stress of the matrix material, $\sigma_{m}$ the macroscopic mean stress and $f$ is the void volume fraction (also called porosity), defined as the average ratio of the void volume to the total volume of the material. The damage parameters $q_{1}$ and $q_{2}$, originally equal to 1.0 in the initial Gurson model, are usually set to $q_{1}=1.5$ and $q_{2}=1.0$ allowing the continuum model to be in good agreement with the localization strain for cell analysis [3]. The extended Gurson model used for the simulations has been implemented by Guzmán [9], featuring Hill '48 type anisotropy of the matrix coupled with a mixed hardening law [6] and classical nucleation and coalescence laws proposed in the GTN model. The evolution of voids is additevely decomposed in the nucleation $\left(f_{n}\right)$ and growth part $\left(f_{g}\right)$, hence:

$$
\dot{f}=\dot{f}_{n}+\dot{f}_{g}=\frac{f_{N}}{S_{N} \sqrt{2 \pi}} \exp \left[-\frac{1}{2}\left(\frac{\epsilon_{M}^{P}-\epsilon_{N}}{S_{N}}\right)^{2}\right] \dot{\epsilon}_{M}^{P}+(1-f) \operatorname{tr} \dot{\epsilon}_{i j}^{p}
$$


with $\epsilon_{M}^{P}$ as the equivalent plastic strain in the matrix and $f_{N}, S_{N}, \epsilon_{N}$ as material parameters. Coalescence is determined by the effective porosity $f^{*}$ function:

$$
f^{*}= \begin{cases}f & \text { if } f<f_{c r} \\ f_{c r}+\frac{\frac{1}{q_{1}}-f_{c r}}{f_{F}-f_{c r}}\left(f-f_{c r}\right) & \text { if } f>f_{c r}\end{cases}
$$

where $f_{c r}$ and $f_{F}$ are material parameters.

\section{Identification of material parameters}

The experiments are performed on a bulk Ti-6AL-4V alloy, depicted in Fig. 1(a). The elasto-viscoplastic behavior of this bulk alloy has been previously characterized by Tuninetti and Habraken [10] and Tuninetti et al. [7].

Plasticity characterization. The tensile tests were performed in LD direction until fracture using universal testing machine and a three 3D optical strain measuring systems (DIC Limess), allowing obtaining the local strain within the localized zone of the specimens and, consequently, the true stresstrue strain curves even after the onset of necking. These data have been used in order to obtain a new set of parameters of the Voce hardening law for the reference direction LD of the CPB06 model (Table 1). The former hardening parameters used in Tuninetti and Habraken [10] and Tuninetti et al. [7] were identified using stress-strain curves until 0.1 axial strain leading to lower values of the axial tensile stress after the onset of necking compared with the experimental data, and therefore lower values of the load in specimen subjected to axial load. Fig. 1(b) shows the results using the old set of parameters (Voce 0.1) and the new ones (Voce 0.2).

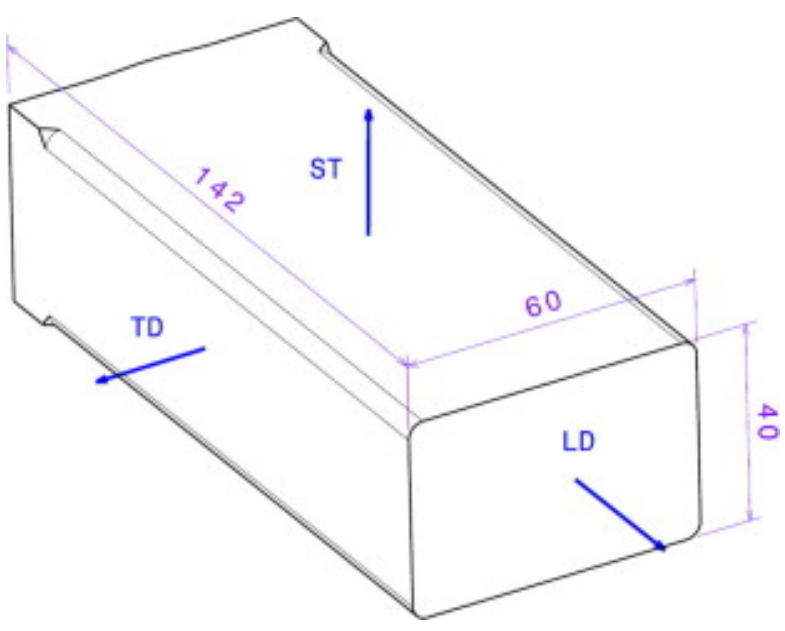

(a)

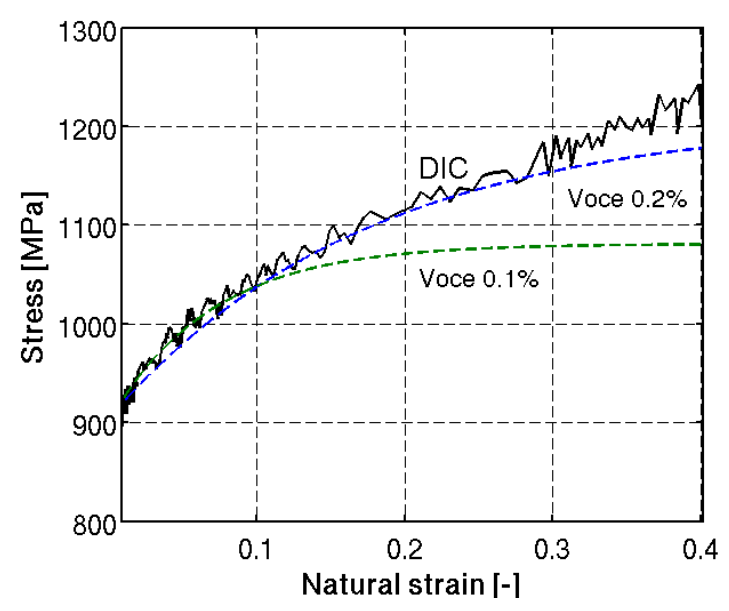

(b)

Fig. 1: (a) Material directions of the TI-6AL-4V ingot (dimensions in [mm]). Longitudinal (LD), Transverse (TD) and Short Transverse (ST) directions. (b) Hardening curve for the TI-6AL-4V, showing the newly identified Voce law until 0.2 axial strain using the DIC curve.

Table 1: New Voce-type of hardening material parameters for the Ti-6AL-4V.

$918.0 \quad 5.8 \quad 290.0$

The anisotropy of the material is described by measuring the flow stress under different stress states and work hardening levels. The anisotropy parameters for the Hill ' 48 model are shown in Table 2, 
while for CPB06 they have been reported previously [10]. Evaluating these two models by looking at their analytical prediction in the stress space (Fig. 2), clear differences appear for the plane stress biaxial and compression states.

Table 2: Anisotropic parameter of the initial Hill '48 yield locus for $W_{p}=1.857\left[\mathrm{~J} \mathrm{~cm}^{-3}\right]$, identified from tensile data in the three orthogonal material directions (LD, TD and ST) and the simple shear in LD-ST plane.

\begin{tabular}{cccccc}
\hline$H$ & $F$ & $G$ & $N$ & $L$ & $M$ \\
1.017 & 0.958 & 0.983 & 3.278 & 3.278 & 3.278 \\
\hline
\end{tabular}

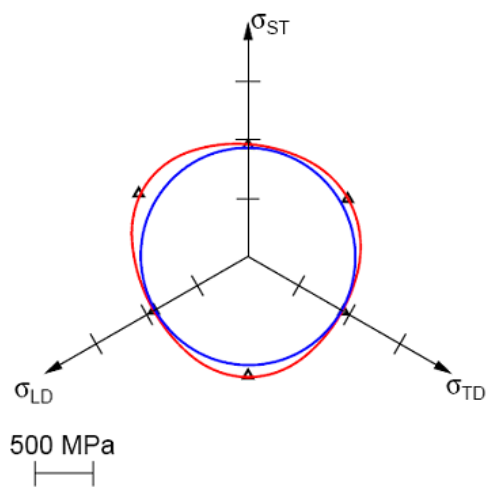

(a) $\pi$-plane at $W_{p 1}$.

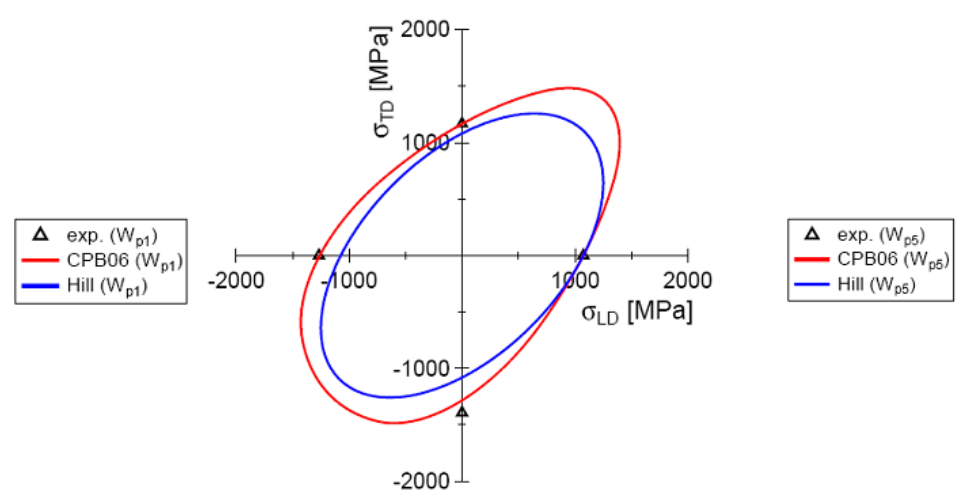

(b) LD-TD plane at $W_{p 5}$.

Fig. 2: Comparison between initial $\left(W_{p 1}=1.857\left[\mathrm{~J} \mathrm{~cm}^{-3}\right]\right)$ and final yield surfaces $\left(W_{p 5}=1.857\left[\mathrm{~J} \mathrm{~cm}^{-3}\right]\right)$ defined by Hill ' 48 and CPB06 at different planes in the stress space.

GTN identification. The identification of the GTN model parameters does not follow the same methodology of the previous plasticity models. The nature of the model, mixing macroscopic and microscopic variable, implies that the search of parameters does not rely in a single approach. Moreover, the use of a optimization algorithm could be an ineffective tool due to the appearance of several local roots. As first step towards a correct identification of the model based on notched tensile tests, the set of parameters of Table 3 was found to be in good agreement with the experimental results. Different microscopic measurements by optical microscopy and tomography confirm that initial porosity is very low $\left(f_{0}<1.0 \times 10^{-6}\right)$.

Table 3: Set of parameters used for the GTN model.

\begin{tabular}{ccccccc}
\hline & $f_{0}$ & $f_{N}$ & $S_{N}$ & $\epsilon_{N}$ & $f_{c r}$ & $f_{F}$ \\
coa1 & 0.0 & 0.04 & 0.10 & 0.30 & 0.03 & 0.04 \\
coa2 & 0.0 & 0.04 & 0.10 & 0.30 & 0.04 & 0.06 \\
\hline
\end{tabular}

\section{Simulations}

Finite element (FE) simulations are performed using the updated Lagrangian FE code Lagamine developed by the ArGEnCo Department of the University of Liège. The 8-node 3D brick element, called BWD3D [11], with a mixed formulation adapted to large strains and large displacements is selected. It involves a reduced integration scheme (with only one integration point) and an hourglass control technique. The three models are compared on three different tests: tensile test on notched specimens with radius $R=1.5[\mathrm{~mm}]$ and $R=5.0[\mathrm{~mm}]$ [7], plus a tensile test in a specimen with a central hole of $R=2.0[\mathrm{~mm}][10]$. The results are shown in Fig. 3. 


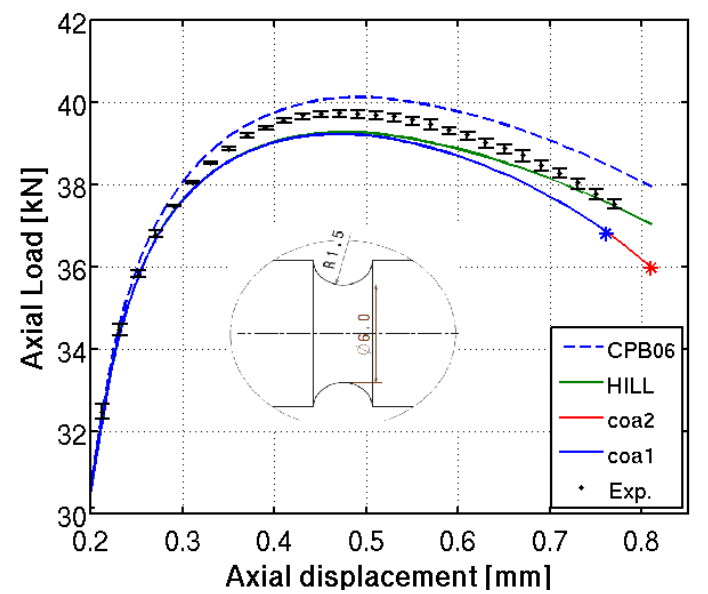

(a) Notch $R=1.5[\mathrm{~mm}]$.

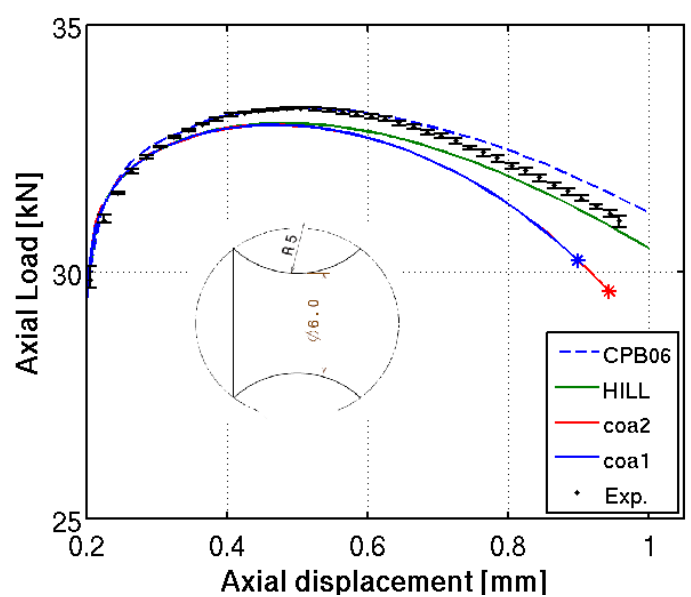

(b) Notch $R=5.0[\mathrm{~mm}]$.

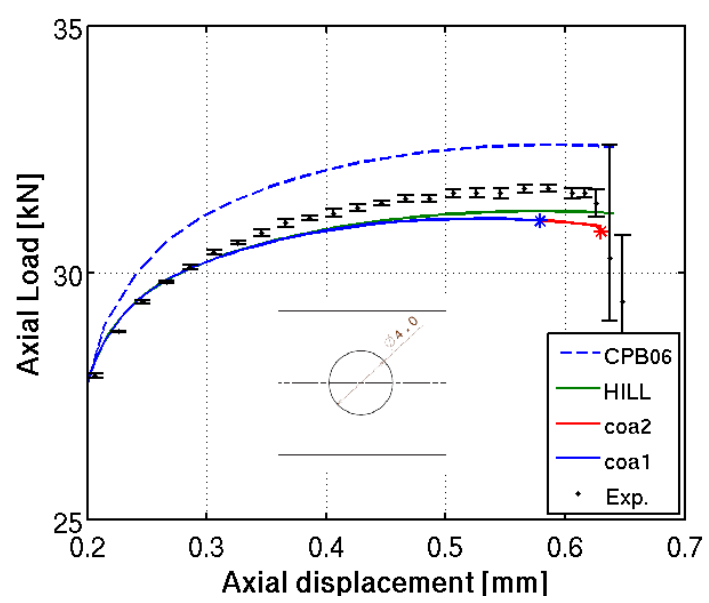

(c) Specimen with a hole.

Fig. 3: Comparison of the three different models with experimental results, for three different geometries. The stars at the end of the curves indicate the onset of coalescence and experimental scattering is expressed by error bars. 
Broadly, the three models gives acceptable predictions, with the CPB06 model slightly overpredicting the force. As underlined in Tuninetti et al. [7], the FE prediction with CPB06 is quite sensitive to its identification methodology. The data set used here (CPB06 identification 4 in Tuninetti et al. [7]) provides optimal notch results but is less adapted for the case of the specimen with the central hole, where more shear is present. Hill ' 48 predictions are better, reaching force values which are in close agreement with experiments. Looking at the damage set of parameters coa1 and coa2, the force reduction is more affected in the notched specimens, in particular $R=5.0[\mathrm{~mm}]$. In the other side, the specimen with a hole is less affected by the damage development giving almost the same results as Hill '48. In the latter, and even if the damage development is not noticeable, coa2 data set gives a good prediction of the onset of coalescence. This data set is able to predict correctly the onset of coalescence for the notched specimen, but for an underestimated force. Coal data set is more accurate predicting the onset of coalescence for the notched specimen $R=1.5[\mathrm{~mm}]$, but also for an underestimated force.

\section{Conclusions}

In this article, a comparison between two plasticity models (Hill '48 and CPB06) and the GTN damage model has been done. From the analyzed specimens, it is clear that a certain amount of damage is being accumulated leading to ductile fracture. The fracture prediction, despite not being perfect, gives a hint of the importance of void nucleation mechanism leading to a ductile fracture. Despite that a single set of parameters matching all the experiments was not possible to obtain, material parameter variation is not severe and the sets should be regarded within a confidence interval. Future work in this topic will be focused in a deeper micromechanical review of the material, plus a more precise characterization of the damage parameters of the GTN model. This also involves damage characterization at low triaxialities or shear-type loads, which is a trending topic in recent years.

\section{References}

[1] J. Lemaitre, Journal of Engineering Materials and Technology 107, 83 (1985).

[2] A. L. Gurson, Journal of Engineering Materials and Technology 99, 2 (1977).

[3] V. Tvergaard, Advances in Applied Mechanics, J. W. Hutchinson, T. Y. Wu, eds. (Elsevier, 1989), vol. 27 of Advances in Applied Mechanics, pp. 83-151.

[4] A. A. Benzerga, J. Besson, European Journal of Mechanics - A/Solids 20, 397 (2001).

[5] R. Hill, Proceedings of the Royal Society A: Mathematical, Physical and Engineering Sciences 193, 281 (1948).

[6] M. Ben Bettaieb, X. Lemoine, L. Duchêne, A. M. Habraken, International Journal for Numerical Methods in Engineering 85, 1049 (2011).

[7] V. Tuninetti, G. Gilles, O. Milis, T. Pardoen, A. Habraken, International Journal of Plasticity 67, 53 (2015).

[8] O. Cazacu, B. Plunkett, F. Barlat, International Journal of Plasticity 22, 1171 (2006).

[9] C. F. Guzmán, Extension of the Gurson model for the LAGAMINE code, Tech. rep., University of Liège, Liège (2014).

[10] V. Tuninetti, A. M. Habraken, Materials Science and Engineering: A 605, 39 (2014).

[11] L. Duchêne, F. El Houdaigui, A. M. Habraken, F. Elhoudaigui, International Journal of Plasticity 23, 1417 (2007). 